\title{
Maladie de Creutzfeldt-Jakob iatrogène et transfusion sanguine
}

La maladie de Creutzfeldt-Jakob (MCJ) est une encéphalopathie subaiguë spongiforme provoquée par des agents transmissibles non conventionnels ou prions dont la nature reste inconnue à ce jour $(\mathrm{m} / \mathrm{s}$ $n^{\circ} 1$, vol. $\left.6, p .77\right)$. La présence des agents transmissibles dans le sang a été décrite dans la MCJ sporadique (la forme humaine la plus fréquente avec près de $90 \%$ des cas) et, dans les modèles animaux d'encéphalopathie subaiguë spongiforme transmissible, cette "virémie " est observée tout au long de la maladie, notamment pendant la phase cliniquement silencieuse qui peut atteindre trentecing ans chez l'homme. Les études épidémiologiques n'ont retrouvé aucun lien statistique entre des antécédents de transfusion sanguine et le risque de développer une $\mathrm{MCJ}$ : il semblerait donc, en gardant en mémoire les difficultés d'interprétation de données concernant une maladie rare (environ un nouveau cas par an et par million d'habitants), dont la période d'incubation est extrêmement longue, qu'il n'existe pas de risque transfusionnel particulier lié à des donneurs de sang en période d'incubation silencieuse de MCJ sporadique [1]. Ces études ne prennent pas en compte les formes iatrogènes de MCJ qui étaient rarissimes jusqu'à ces dernières années. Toutefois, le traitement de près de 1500 femmes en Australie par des lots d'hormones gonadotrophiques potentiellement contaminées par des prions, et de près de 1000 enfants en France par des lots d'hormone de croissance suspects, implique un risque non négligeable de don de sang potentiellement contaminé. Les contaminations par voie périphérique (injections souscutanées, intramusculaires ou intraveineuses) entraînent des MCJ iatrogènes caractérisées par une présentation clinique tout à fait particulière: le syndrome cérébelleux est majeur tandis que l'atteinte frontale est mineure, voire absente, alors que, dans les formes sporadiques de MC.J, le syndrome démentiel est très souvent prédominant [2].

En Australie, quatre personnes ont développé une MCI de type iatrogène par contamination périphérique, cing ans après une transfusion sanguine dans une région où des femmes, traitées par des hormones gonadotrophiques potentiellement contaminées, étaient donneuses de sang; l'une d'entre elles a développé dans l'intervalle une MCJ iatrogène. D'après les auteurs, il pourrait s'agir des premiers cas observés de transmission de MCJ iatrogène par transfusion sanguine [3].

En France, trente et un malades ont développé à ce jour une MCI iatrogène après traitement par hormone de croissance extractive. Nous avons pu étudier les caractéristiques moléculaires de quatre cas de MCJ iatrogène et de sept cas de MCJ sporadique [4]. Sur des échantillons prélevés en postmortem, nous avons étudié dans le cervelet et le lobe frontal la répartition de la PrPCJD, l'isoforme anormale de la protéine du prion (PrP), qui s'accumule dans le cerveau parallèlement au développement de la maladie. Les malades décédés de MCJ iatrogène avaient des quantités importantes de PrPCJD dans le cervelet et des quantités faibles dans le lobe frontal. Une répartition inverse a été retrouvée dans les échantillons des sept cas de MCJ sporadique étudiés, indépendamment des signes cliniques initiaux. Cette différence de distribution correspond exactement au tableau clinique majoritairement cérébelleux ou démentiel des deux groupes de MCJ [4]. Ce résultat peut être lié à la génétique de l'hôte, à la voie d'inoculation ou à la sélection de souches particulières de prions. Le gène de la PrP semble constituer le principal gène de susceptibilité à ces maladies et nous n'avons retrouvé aucune différence génétique chez les malades atteints de MCJ iatrogène et sporadique [5]. Dans les modèles expérimentaux, la voie d'inoculation, tout comme la dose infectieuse, ne modifie pas notablement la répartition de la PrP anormale au stade terminal de la maladie. En l'état actuel des connaissances, les résultats rapportés semblent donc impliquer la sélection de souches particulières de prions [4]. Cette hypothèse est renforcée par la transmission de la maladie à un hamster inoculé par voie intra-cérébrale avec des globules blancs du premier cas français de MCJ iatrogène : les lésions cérébrales observées étaient uniquement retrouvées dans le cervelet et le tronc cérébral, suggérant un tropisme similaire à celui observé chez les patients.

De telles souches, qui seraient sélectionnées après inoculation par 
des voies peu efficaces (par rapport à la voie intra-cérébrale), pourraient théoriquement présenter une virulence particulière, notamment au niveau périphérique, dans le sang et également dans les tissus susceptibles de servir de greffon. Dans cette hypothèse, les données épidémiologiques actuelles, concernant l'absence de risque transfusionnel lié aux cas de MCJ sporadique, ne peuvent être étendues aux cas de MCJ iatrogène. Une mesure préventive a déjà été prise dans les collectes en France, avec l'exclusion des dons de sang provenant de patients antérieurement traités par l'hormone de croissance.

J.P.D. Creutzfeldt-Jakob disease by blood transfusion. C.L Lancel 1993; $341: 768$

C.L. 4. Deslys JP, Lasmézas C, Dormont D. SelecD.D. tion of specific strains in iatrogenic Creuzfeldt-Jakob disease. Lancet $1994 ; 343$ : 848-9.

5. Deslys JP, Marcé D, Dormont D. Similar genetic susceptibility in iatrogenic and sporadic Creutzfeldt-Jakob disease. J Gen Virol $1994 ; 75: 23-7$.
1. Esmonde TFG, Will RG, Slattery JM Knight R, Harriesjones R, Desilva R, Matthews
WB. Creutzfeldt-Jakob disease and blood

fire in Medicine - MA, Will RG. Friendly and Creutzfeldt-Jakob disease. Lancet 1992 ;
Le taxol ralentit la progression de la polykystose rénale congénitale. La polykystose rénale congénitale (PRC) est la plus fréquente des maladies héréditaires des reins, fournissant $10 \%$ des malades qui nécessitent épuration artificielle et transplantation rénale. La culture primaire de cellules tubulaires rénales provenant de malades PRC a montré qu'elles forment spontanément des kystes in vitro. Des inhibiteurs de la synthèse protéique ( $\alpha$-amanitine) ou des antimétabolites tels le méthotrexate et la cytosine arabinoside n'empêchent pas la transformation kystique; en revanche, les inhibiteurs de transports ioniques (ouabaine et amiloride) l'inhibent de façon réversible ainsi que les promoteurs de l'assemblage des microtubules (taxol, colchicine, vinblastine), suggérant un rôle des mouvements liquidiens transépithéliaux et de l'architecture du cytosquelette dans la formation des kystes. La mise au point d'un modèle murin, la souris $c p k$, qui développe une polykystose rénale récessive conduisant à la mort par insuffisance rénale en 4 à 5 semaines, a permis de mieux comprendre la maladie et de tester de nouvelles voies thérapeuti- ques. In vivo, le taxol administré par voie intrapéritonéale une fois par semaine semble arrêter la progression de la polykystose : à $200 \mathrm{j}$ les souris ont une taille et un poids normaux, et une créatininémie normale [1]. Les autres antimitotiques testés ont été sans effet. Comment agit le taxol? Il favorise l'assemblage des microtubules qui participent au trafic intracellulaire des vésicules, à l'exocytose et à l'endocytose, et au mouvement entre réticulum endoplasmique et appareil de Golgi. Il est possible que des anomalies de fonctionnement cellulaire liées aux microtubules soient en cause dans la localisation apicale aberrante de la $\mathrm{Na}+, \mathrm{K}+$ ATPase dans les tubules rénaux de $\mathrm{PRC}$, et que le taxol corrige ce défaut. Les résultats rapportés dans cette étude de PRC indiquent que le réseau microtubulaire est sans doute impliqué dans la formation des kystes dans le modèle murin, et donnent un espoir de voir traiter cette grave maladie humaine dont on ne connaît pas encore le ou les gènes responsables.

[1. Woo DDL, et al. Nature 1994; 368 : 750-3.] $\square$ Des mutations dans les gènes de Fas et du ligand de Fas causent, chez la souris, un syndrome lymphoprolifératif généralisé. Nous avons déjà rappelé plusieurs fois que le phénotype $l p r$ (lymphoproliferation) était dû à une mutation homozygote du gène codant pour la protéine Fas, un récepteur membranaire dont la stimulation entraîne l'apoptose $(\mathrm{m} / \mathrm{s}$ $n^{\circ} 11$, vol. 9, p. 1279). Le ligand de Fas est une protéine appartenant à la famille TNF (tumor necrosis factor), $\left(m / s n^{\circ} 2\right.$, vol. $\left.10, p .234\right)$. Le gène codant pour ce ligand (Fasl) a été cloné. Une équipe japonaise d'Osaka, dirigée par Nagata, démontre maintenant que la mutation du gène Fasl entraîne le phénotype murin gld (generalized lymphoproliferative disease), très proche du phénotype $l p r$, caractérisé comme lui par une lymphoadénopathie généralisée et des manifestations auto-immunes [1]. L'importance du signal passant par Fas est donc attestée par la symptomatologie associée à des mutations modifiant le ligand et le récepteur de ce système.

[1. Takahashi T, et al. Cell 1994; 76: 969-76.] 